\title{
THE OPTICAL SOCIETY OF INDIA
}

\author{
CORPORATE MEMBERS
}

1. Central Scientific Instruments Organisation Sector-30, Chandigarh-16 020.

2. Harvin Optical and Glass Industries, C-34 Industrial Estate, Sanatnagar, Hyderabad-18.

3. Govt. of India Ministry of Defence,

$\mathrm{R}$ \& D Organisation

I.R.D.E., Dehra Dun-248 008.

4. Bharat Ophthalmic Glass Ltd., (A Govt. of India Undertaking),

Ophthalmic Glass Plant, Lenin Sarani, Durgapur-10, W.B.

5. National Physical Laboratory.

New Delhi-12.

6. Indian Institute of Technology,

New Delhi-29.

7. Precision Optics and Machineries (P) Ltd., P-26 Grahams Land, Calcutta-40.

8. National Instruments Ltd., 1/1 Raja S. C. Mullick Road, Calcutta-700 032.

9. Vacuum Instruments Company, 81 Reghar Pura, New Delhi-110 005.

10. Spectralase, 94, Bhagat Singh Market, New Delhi-110 001.

11. Survey of India, Geodetic and Research Branch, Post Box No. 77, Dehra Dun.

12. M/S Spectra Systems (P)Ltd., MSR Industrial Estate, Gokula Extn, Bangalore-560 04.

13. Crafts De Fluorescent, Plot 221, Goregaon Mulunal Link Road, Off. L-B Shastri Marg, Bhandup. Bombay-400078.
14. Peico Electronics and Electricals Limited, Band Box Building, 254-D, Dr. Annie Besant Road, Bombay-400025.

15. Fie Research Institute. Ganganagar P. O. Ichalkaranji, Pin : 416116, Maharashtra.

16. General Optics (Asia) Ltd.

R. S. No. 84/1, Nallavadu Road, Thavalakuppam Pondicherry 605007.

17. Indian Institute of Astrophysics, Bangalore-560034.

18. Isro Headquarters, F. Block, Cauvery Bhavan, Kempagowda Road, Bangalore-560 009.

19. The Oriental Science Apparatus Workshops, Jawaharlal Nehru Marg., Ambala Cantt-133 001, Haryana.

20. Harvin Agencies, 6-3-1090/B/4, Rajbhavan Rd., Samajiguda, Hyderabad- 500482.

21. OPTIRBGION, 13/28. Shaki Nagar, Delhi-110007.

22. Central Glass and Ceramic Research Institute, P. O. Jadavpur University, Calcutta-700 032.

23. Indian Electrics Private Limited 96 Kothawal chavadi Street Madras-600 015.

22. RAAS Corporation 60 Basant Lok Vasant Vihar New Delhi-110 057

25. Metallurgical \& Engineering Consultants (India) Ltd. Ranchi-834002, Bihar.

26. Hindustan Cables Ltd. P. O. Hindustan Cables Hyderabad-500 051, A. P. 